INPLASY

PROTOCOL

To cite: Chen et al. Efficacy of acupuncture therapy on cancer-related-insomnia: A Protocol for Systematic Review and Network Metaanalysis. Inplasy protocol 202210095. doi: 10.37766/inplasy2022.1.0095

Received: 18 January 2022

Published: 18 January 2022

Corresponding author: Liying Chen

chenlychris@163.com

Author Affiliation:

Zhejiang Chinese Medical

University.

Support: NA.

Review Stage at time of this submission: Preliminary searches.

Conflicts of interest: None declared.

\section{Efficacy of acupuncture therapy on cancer-related-insomnia: A Protocol for Systematic Review and Network Meta-analysis}

Chen, LY1; Fu, CW2; Wu, YB3; Xu, ST4; Lin, XM55.

Review question / Objective: Efficacy of various acupuncture therapies in treating cancer-related-insomnia.

Condition being studied: Cancer-related-insomnia (CRI) is a kind of secondary insomnia, which has a high prevalence of about $35-70 \%$ in cancer survivors. CRI often has a negative impact on the quality of life, slowing the recovery of cancer survivors, causing depression, fatigue, and even memory loss. However, current medication has not met the satisfaction. Therefore, cancer survivors often shift their attention to complementary and alternative medicine(CAM). Among all, acupuncture therapy is a promising CAM which has been widely used in cancer survivors. Several meta-analyses have demonstrated the effectiveness of acupuncture therapies in treating CRI. However, it is still unclear which acupuncture regimen is the best choice. Thus, by conducting network meta-analysis, we will provide suggestion for clinical practice of using acupuncture in CRI.

INPLASY registration number: This protocol was registered with the International Platform of Registered Systematic Review and Meta-Analysis Protocols (INPLASY) on 18 January 2022 and was last updated on 18 January 2022 (registration number INPLASY202210095).

\section{INTRODUCTION}

Review question / Objective: Efficacy of various acupuncture therapies in treating cancer-related-insomnia.

Condition being studied: Cancer-relatedinsomnia (CRI) is a kind of secondary insomnia, which has a high prevalence of about $35-70 \%$ in cancer survivors. CRI often has a negative impact on the quality of life, slowing the recovery of cancer survivors, causing depression, fatigue, and even memory loss. However, current medication has not met the satisfaction. Therefore, cancer survivors often shift their attention to complementary and alternative medicine(CAM). Among all, acupuncture 
therapy is a promising CAM which has been widely used in cancer survivors. Several meta-analyses have demonstrated the effectiveness of acupuncture therapies in treating CRI. However, it is still unclear which acupuncture regimen is the best choice. Thus, by conducting network metaanalysis, we will provide suggestion for clinical practice of using acupuncture in CRI.

\section{METHODS}

Participant or population: Cancer survivors with cancer-related-insomnia

Intervention: Various acupuncture therapies including acupuncture, moxibustion, acupressure, electroacupuncture, catgut embedding, transcutaneous electric acupoint stimulation, auriculotherapy and so on.

Comparator: Sham acupuncture or medicine or other interventions.

Study designs to be included: Randomized control trails.

Eligibility criteria: Peer-reviewed randomized control trails will be eligible for inclusion. And language will be restricted to English and Chinese.

Information sources: Eight electronic databases will be searched from set up to March 1, 2022 including PubMed, Cochrane library, Web of Science, Embase, China National Knowledge Infrastructure, Wanfang Database, VIP Database, and China Biology Medicine.

Main outcome(s): The Pittsburgh Sleep Quality Index(PSQI)

Additional outcome(s): 1. Other CRI-related questionnaires; 2. Negative emotion questionnaires; 3. Pain questionnaires; 4. Fatigue questionnaires; 5. Quality of life questionnaires.

Quality assessment / Risk of bias analysis: We will use Cochrane risk-of-bias tool
(ROB 2.0) to evaluate the quality of included studies.

Strategy of data synthesis: Pairwise analysis will be performed by STATA and Network meta-analysis by OpenBUGS, $R$ and STATA. Severally, we will present continuous and binary outcomes in terms of mean differences and hazard ratios, with corresponding $95 \%$ confidence intervals. In pairwise meta-analyses, heterogeneity can be assessed by Higgins' I-square statistic, a fixed-effects model will be used if Isquare value is $<50 \%$. Both global and local inconsistencies will be evaluated by STATA. $R$ will be applied to represent statistical heterogeneity. Finally, league figure and surface under the cumulative ranking curve will be carried out by OpenBUGS.

Subgroup analysis: Subgroup analyses and regression analyses will be performed based on the heterogeneity and the similarity of the acupuncture therapy types and clinical differences.

Sensitivity analysis: Before selecting model, a sensitivity analysis will be carried out if necessary and there are adequate studies.

Language: Chinese and English.

Country(ies) involved: China.

Keywords: Cancer-related-insomnia; Acupuncture therapy; Network Metaanalysis.

Contributions of each author:

Author 1 - Liying Chen.

Email: chenlychris@163.com

Author 2 - Chengwei Fu.

Email: 405417101@qq.com

Author 3 - Boyu Wu.

Email: 1225571819@qq.com

Author 4 - Shiting Xu.

Email: 512918907@qq.com

Author 5 - Xianming Lin.

Email: linxianming66@126.com 